\title{
Local stable manifold of Langevin differential equations with two fractional derivatives
}

\author{
JinRong Wang ${ }^{1 *}$, Shan Peng ${ }^{1 *}$ and D O'Regan ${ }^{2 *}$
}

\section{"Correspondence:}

wjr9668@126.com

pengshanxing@126.com;

donal.oregan@nuigalway.ie

'Department of Mathematics,

Guizhou University, Guiyang,

Guizhou 550025, P.R. China

${ }^{2}$ School of Mathematics, Statistics

and Applied Mathematics, National

University of Ireland, Galway, Ireland

\section{严 Springer}

\begin{abstract}
In this paper, we investigate the existence of local center stable manifolds of Langevin differential equations with two Caputo fractional derivatives in the two-dimensional case. We adopt the idea of the existence of a local center stable manifold by considering a fixed point of a suitable Lyapunov-Perron operator. A local center stable manifold theorem is given after deriving some necessary integral estimates involving well-known Mittag-Leffler functions.
\end{abstract}

MSC: 26A33; 34A34; 34D35

Keywords: local stable manifolds; Langevin differential equations; Mittag-Leffler functions

\section{Introduction}

Fractional calculus was introduced by Liouville and Riemann. The concept of non-integer calculus is a generalization of the traditional integer-order calculus that was mentioned by Leibniz and L'Hospital. Fractional calculus is a rapidly growing area with many applications in diverse fields ranging from physical sciences, engineering to biological sciences and economics.

Fractional mathematical modeling and fractional differential equations theory arise naturally in applications; see [1-7] and the references given therein. Fractional Langevin equations (i.e., equations involving two fractional derivatives with fractional initial conditions) are used to describe stochastic problems in physics, chemistry and electrical engineering, and the existence and stability results for these equations were considered in [8-23] and the references given therein.

In [24] the authors gave a local stable manifold theorem near a hyperbolic equilibrium point for planar fractional differential equations by considering the Lyapunov-Perron operator via the asymptotic behavior of the Mittag-Leffler function. In [25] a reliable strategy to approximate the local stable manifold near a hyperbolic equilibrium point for nonlinear fractional differential systems is presented and, using the fractional Hartman-Grobman theorem, the local behavior near a hyperbolic equilibrium point is investigated. In [26] a local center manifold result for fractional ordinary differential equations is given. In the literature stable manifold results for fractional Langevin equations are still very limited. In

(c) The Author(s) 2017. This article is distributed under the terms of the Creative Commons Attribution 4.0 International License (http://creativecommons.org/licenses/by/4.0/), which permits unrestricted use, distribution, and reproduction in any medium, provided you give appropriate credit to the original author(s) and the source, provide a link to the Creative Commons license, and indicate if changes were made. 
[27] the authors consider center stable manifolds for planar fractional damped equations involving two Caputo fractional derivatives with zero and first derivative initial conditions which act on two-dimensional vectors with one order belonging to $(1,2)$, the other belonging to $(0,1)$.

In this paper, motivated by [14, 16, 24, 25, 27], we study local center stable manifolds of fractional Langevin equations of the type:

$$
\left\{\begin{array}{l}
{ }^{c} \mathcal{D}_{0, t}^{\mu}\left({ }^{c} \mathcal{D}_{0, t}^{v} x(t)\right)+\mathcal{A} x(t)=h(x(t), t), \quad t \geq 0, \\
x(0)=\mathbf{0}=(0,0)^{T}, \quad\left[{ }^{c} \mathcal{D}_{0, t}^{v} x(t)\right]_{t=0}=\bar{x}=\left(x_{3}, x_{4}\right)^{T},
\end{array}\right.
$$

where ${ }^{c} \mathcal{D}_{0, t}^{\mu}$ and ${ }^{c} \mathcal{D}_{0, t}^{v}$ denote the Caputo fractional derivative of order $\mu, v \in(0,1)$ with the lower limit zero (see Definition 1.1), $0<\mu+\nu<1$ and $\mathcal{A}=\operatorname{diag}\left(\lambda_{1}, \lambda_{2}\right)$ with $\lambda_{1}>0, \lambda_{2}<0$ and $h(x, t)=\left(h_{1}(x, t), h_{2}(x, t)\right)^{T}$. The function $h: \mathbb{R}^{2} \times \mathbb{R}_{+} \rightarrow \mathbb{R}^{2}$ satisfies

$$
\|h(x, t)-h(y, t)\| \leq l_{h}(r)\|x-y\|
$$

for all $\|x\|,\|y\| \leq r$ with $h(0, t)=0, \lim _{r \rightarrow 0} l_{h}(r)=0$, where $\|\cdot\|=\|(\cdot, \cdot)\|=\max \{|\cdot|,|\cdot|\}$. Note for (1), two different order derivative are involved, and from [17, Theorem 3.3] we can rewrite ${ }^{c} \mathcal{D}_{0, t}^{\mu}\left({ }^{c} \mathcal{D}_{0, t}^{v} x(t)\right)$ as ${ }^{c} \mathcal{D}_{0, t}^{\mu+v} x(t)$.

Definition 1.1 (see [1]) The Caputo derivative of order $\gamma$ for a function $\varpi:[0, \infty) \rightarrow \mathbb{R}$ can be written as ${ }^{c} \mathcal{D}_{0, t}^{\gamma} \varpi(t)={ }^{R L} \mathcal{D}_{0, t}^{\gamma}\left(\varpi(t)-\sum_{k=0}^{n-1} \frac{t^{k}}{k !} \varpi^{(k)}(0)\right), t>0, N-1<\gamma<N, N=$ $1,2, \ldots$, where ${ }^{R L} D_{0, t}^{\gamma} \varpi$ denotes the Riemann-Liouville derivative of order $\gamma$ with the lower limit zero for a function $\varpi$, which is given by ${ }^{R L} \mathcal{D}_{0, t}^{\gamma} \varpi(t)=\frac{1}{\Gamma(n-\gamma)} \frac{d^{n}}{d t^{n}} \int_{0}^{t} \frac{\varpi(s)}{(t-s)^{\gamma+1-n}} d s, t>0$, $N-1<\gamma<N, N=1,2, \ldots$

From [16, Lemma 2.8], taking the Laplace transform of the Caputo fractional derivative and the inverse Laplace transform of the functions, the solution $\psi(\cdot, \mathbf{0}, \bar{x})$ of $(1)$ is given by

$$
\begin{aligned}
\psi(t, \mathbf{0}, \bar{x})= & \mathbb{E}_{\mu+v, v+1}\left(-t^{\mu+v} \mathcal{A}\right) t^{\nu} \bar{x} \\
& +\int_{0}^{t}(t-s)^{\mu+v-1} \mathbb{E}_{\mu+v, \mu+v}\left(-(t-s)^{\mu+v} \mathcal{A}\right) h(\psi(s, x, \bar{x}), s) d s .
\end{aligned}
$$

Next, we give the definition of a local center stable manifold.

Definition 1.2 By a local center stable manifold of (1), we mean the set of all small $\bar{x}$ for which the solution of (1) is bounded on $\mathbb{R}_{+}$.

For some certain $\bar{x}$, the solution's limit of (1) is zero when the time variable tends to infinity.

Let $X_{\infty}\left(\mathbb{R}_{+}, Y\right)$ be the Banach space of all continuous functions from $\mathbb{R}_{+}$into a Banach space $Y$ with the norm $\|z\|_{\infty}=\sup \left\{\|z(t)\|_{Y}: t \in \mathbb{R}_{+}\right\}$. We adopt the ideas in [24, 27] and construct a suitable Lyapunov-Perron operator

$$
\mathcal{L P}=\left(\mathcal{L} \mathcal{P}_{1}, \mathcal{L} \mathcal{P}_{2}\right): X_{\infty}\left(\mathbb{R}_{+}, \mathbb{R}^{2}\right) \rightarrow X_{\infty}\left(\mathbb{R}_{+}, \mathbb{R}^{2}\right)
$$


as follows:

$$
\begin{aligned}
\mathcal{L P}_{1}(\eta)(t)= & \mathbb{E}_{\mu+v, v+1}\left(-t^{\mu+v} \lambda_{1}\right) t^{v} x_{3} \\
& +\int_{0}^{t}(t-s)^{\mu+\nu-1} \mathbb{E}_{\mu+v, \mu+v}\left(-(t-s)^{\mu+v} \lambda_{1}\right) h_{1}(\eta(s), s) d s,
\end{aligned}
$$

and

$$
\begin{aligned}
\mathcal{L P}_{2}(\eta)(t)= & -\left(-\lambda_{2}\right)^{\frac{1-\mu}{\mu+\nu}} \mathbb{E}_{\mu+v, v+1}\left(-t^{\mu+v} \lambda_{2}\right) t^{\nu} \int_{0}^{\infty} \exp \left(-\left(-\lambda_{2}\right)^{\frac{1}{\mu+\nu}} s\right) h_{2}(\eta(s), s) d s \\
& +\int_{0}^{t}(t-s)^{\mu+\nu-1} \mathbb{E}_{\mu+v, \mu+\nu}\left(-(t-s)^{\mu+v} \lambda_{2}\right) h_{2}(\eta(s), s) d s .
\end{aligned}
$$

Then we show that the local center stable manifold of (1) can be characterized as a fixed point of the above Lyapunov-Perron operator $\mathcal{L P}$ and the fixed point is bounded.

The rest of this paper is organized as follows. In Section 2, we give some fundamental estimates related to Mittag-Leffler functions, and in Section 3, we present the main result of this paper concerning center stable manifolds. An example is given to demonstrate the application of our main result.

\section{Integral estimates related to Mittag-Leffler functions}

The following explicit estimates of Mittag-Leffler functions are useful in the sequel. One can use the integrable expansion of Mittag-Leffler functions [28, Theorem 2.3] and adopt the ideas in [24, Lemma 3] to derive explicit estimates of Mittag-Leffler functions (for more details, we refer the reader to [29, Lemma 2.5]).

Lemma 2.1 (see [29, Lemma 2.5]) Let $\lambda>0$ be arbitrary. For any $\alpha \in(0,1], \beta \in \mathbb{R}$ and $\beta<1+\alpha$. Denote $m(\alpha, \beta, \lambda)=\max \left\{m_{1}(\alpha, \beta, \lambda), m_{2}(\alpha, \beta, \lambda)\right\}$, where

$$
\begin{aligned}
& m_{1}(\alpha, \beta, \lambda)=\frac{|\sin (\pi \beta)| \int_{0}^{\infty} r^{\frac{1-\beta+\alpha}{\alpha}} \exp \left(-r^{\frac{1}{\alpha}}\right) d r}{\sin ^{2}(\pi \alpha) \pi \alpha \lambda^{2}}, \\
& m_{2}(\alpha, \beta, \lambda)=\frac{|\sin (\pi(\beta-\alpha))| \int_{0}^{\infty} r^{\frac{1-\beta}{\alpha}} \exp \left(-r^{\frac{1}{\alpha}}\right) d r}{\sin ^{2}(\pi \alpha) \pi \alpha \lambda} .
\end{aligned}
$$

(i) For all $t>0$, we have

$$
\begin{aligned}
\left|t^{\beta-1} \mathbb{E}_{\alpha, \beta}\left(-\lambda t^{\alpha}\right)\right| & \leq \frac{m_{1}(\alpha, \beta, \lambda)}{t^{2 \alpha-\beta+1}}+\frac{m_{2}(\alpha, \beta, \lambda)}{t^{\alpha-\beta+1}} \\
& \leq m(\alpha, \beta, \lambda)\left(\frac{1}{t^{2 \alpha-\beta+1}}+\frac{1}{t^{\alpha-\beta+1}}\right) .
\end{aligned}
$$

In particular, we have

$$
\begin{aligned}
& \left|t^{\alpha-1} \mathbb{E}_{\alpha, \alpha}\left(-\lambda t^{\alpha}\right)\right| \leq \frac{m(\alpha, \alpha, \lambda)}{t^{\alpha+1}}, \\
& \left|\mathbb{E}_{\alpha}\left(-\lambda t^{\alpha}\right)\right| \leq \frac{m(\alpha, 1, \lambda)}{t^{\alpha}}
\end{aligned}
$$


(ii) For all $t>0$, we have

$$
\begin{aligned}
\left|t^{\beta-1} \mathbb{E}_{\alpha, \beta}\left(\lambda t^{\alpha}\right)-\frac{1}{\alpha} \lambda^{\frac{1-\beta}{\alpha}} \exp \left(\lambda \frac{1}{\alpha} t\right)\right| & \leq \frac{m_{1}(\alpha, \beta, \lambda)}{t^{2 \alpha-\beta+1}}+\frac{m_{2}(\alpha, \beta, \lambda)}{t^{\alpha-\beta+1}} \\
& \leq m(\alpha, \beta, \lambda)\left(\frac{1}{t^{2 \alpha-\beta+1}}+\frac{1}{t^{\alpha-\beta+1}}\right) .
\end{aligned}
$$

In particular, we have

$$
\begin{aligned}
& \left|t^{\alpha-1} \mathbb{E}_{\alpha, \alpha}\left(\lambda t^{\alpha}\right)-\frac{1}{\alpha} \lambda^{\frac{1-\alpha}{\alpha}} \exp \left(\lambda^{\frac{1}{\alpha}} t\right)\right| \leq \frac{m(\alpha, \alpha, \lambda)}{t^{\alpha+1}}, \\
& \left|\mathbb{E}_{\alpha}\left(\lambda t^{\alpha}\right)-\frac{1}{\alpha} \exp \left(\lambda^{\frac{1}{\alpha}} t\right)\right| \leq \frac{m(\alpha, 1, \lambda)}{t^{\alpha}}
\end{aligned}
$$

Remark 2.2 Note that $m_{1}(\alpha, \beta, \lambda)=0$ if $\beta=1$. Then $m(\alpha, \beta, \lambda)=m_{2}(\alpha, \beta, \lambda)$ if $\beta=1$. The previous results in [24, Lemma 3] are special cases of the above lemma.

Lemma 2.3 For $\lambda>0$, define

$$
P(\mu, v, \lambda)=\max \left\{\sup _{z \in[-\lambda, 0]} \mathbb{E}_{\mu+v, \mu+v+1}(z), \lambda^{\frac{-\mu}{\mu+\nu}} \mathbb{E}_{\mu+v, v+1}(\lambda), \frac{\mathbb{E}_{\mu+v, \mu+v}(\lambda)}{\mu+v}+\lambda^{\frac{-\mu}{\mu+\nu}} \mathbb{E}_{\mu+v, v+1}(\lambda)\right\} .
$$

Then, for any function $g \in X_{\infty}\left(\mathbb{R}_{+}, \mathbb{R}\right)$, the following statements hold for all $t \in[0,1]$ :

(i) $\left|\int_{0}^{t}(t-s)^{\mu+\nu-1} \mathbb{E}_{\mu+\nu, \mu+\nu}\left(-(t-s)^{\mu+v} \lambda\right) g(s) d s\right| \leq P(\mu, v, \lambda)\|g\|_{\infty}$.

(ii) $\left|\lambda^{\frac{1-\mu}{\mu+\nu}} t^{\nu} \mathbb{E}_{\mu+v, v+1}\left(t^{\mu+\nu} \lambda\right) \int_{t}^{\infty} \exp \left(-\lambda^{\frac{1}{\mu+\nu}} s\right) g(s) d s\right| \leq P(\mu, \nu, \lambda)\|g\|_{\infty}$.

(iii) $\mid \int_{0}^{t}\left[(t-s)^{\mu+\nu-1} \mathbb{E}_{\mu+\nu, \mu+\nu}\left((t-s)^{\mu+\nu} \lambda\right)\right.$

$$
\begin{aligned}
& \left.-\lambda^{\frac{1-\mu}{\mu+\nu}} t^{\nu} \mathbb{E}_{\mu+\nu, v+1}\left(t^{\mu+\nu} \lambda\right) \exp \left(-\lambda \frac{1}{\mu+\nu} s\right)\right] g(s) d s \mid \\
\leq & P(\mu, v, \lambda)\|g\|_{\infty} .
\end{aligned}
$$

Proof

(i) Using the fact $\int_{0}^{t}(t-s)^{\mu+\nu-1} \mathbb{E}_{\mu+\nu, \mu+\nu}\left(-(t-s)^{\mu+\nu} \lambda\right) d s=t^{\mu+\nu} \mathbb{E}_{\mu+\nu, \mu+v+1}\left(-\lambda t^{\mu+\nu}\right)$, and noticing that Mittag-Leffler functions are increasing functions on $[0, \infty)$, we have

$$
\begin{aligned}
& \left|\int_{0}^{t}(t-s)^{\mu+\nu-1} \mathbb{E}_{\mu+v, \mu+\nu}\left(-(t-s)^{\mu+v} \lambda\right) g(s) d s\right| \\
& \quad \leq t^{\mu+\nu} \mathbb{E}_{\mu+v, \mu+v+1}\left(-\lambda t^{\mu+\nu}\right)\|g\|_{\infty} \leq \sup _{z \in[-\lambda, 0]} \mathbb{E}_{\mu+v, \mu+v+1}(z)\|g\|_{\infty} .
\end{aligned}
$$

(ii) In the same way, we have

$$
\begin{aligned}
& \left|\lambda^{\frac{1-\mu}{\mu+v}} t^{\nu} \mathbb{E}_{\mu+v, v+1}\left(t^{\mu+v} \lambda\right) \int_{t}^{\infty} \exp \left(-\lambda^{\frac{1}{\mu+\nu}} s\right) g(s) d s\right| \\
& \quad \leq \lambda^{\frac{1-\mu}{\mu+\nu}} \mathbb{E}_{\mu+\nu, v+1}(\lambda) \int_{0}^{\infty} \exp \left(-\lambda^{\frac{1}{\mu+\nu}} s\right) d s\|g\|_{\infty} \leq \lambda^{\frac{-\mu}{\mu+\nu}} \mathbb{E}_{\mu+v, v+1}(\lambda)\|g\|_{\infty} .
\end{aligned}
$$


(iii) Similarly, we derive

$$
\begin{aligned}
& \mid \int_{0}^{t}\left[(t-s)^{\mu+v-1} \mathbb{E}_{\mu+v, \mu+v}\left((t-s)^{\mu+v} \lambda\right)\right. \\
& \left.-\lambda^{\frac{1-\mu}{\mu+\nu}} t^{\nu} \mathbb{E}_{\mu+v, v+1}\left(t^{\mu+v} \lambda\right) \exp \left(-\lambda^{\frac{1}{\mu+\nu}} s\right)\right] g(s) d s \\
& \leq \int_{0}^{t}\left[(t-s)^{\mu+\nu-1} \mathbb{E}_{\mu+\nu, \mu+\nu}(\lambda)+\lambda^{\frac{1-\mu}{\mu+\nu}} \mathbb{E}_{\mu+\nu, v+1}(\lambda) \exp \left(-\lambda^{\frac{1}{\mu+\nu}} s\right)\right] d s\|g\|_{\infty} \\
& =\left[\frac{1}{\mu+v} t^{\mu+v} \mathbb{E}_{\mu+v, \mu+v}(\lambda)\right. \\
& \left.+\lambda^{\frac{1-\mu}{\mu+\nu}} \mathbb{E}_{\mu+v, v+1}(\lambda)\left(-\lambda^{\frac{-1}{\mu+v}} \exp \left(-\lambda^{\frac{1}{\mu+v}} t\right)+\lambda^{\frac{-1}{\mu+v}}\right)\right]\|g\|_{\infty} \\
& \leq\left[\frac{\mathbb{E}_{\mu+v, \mu+v}(\lambda)}{\mu+v}+\lambda^{\frac{-\mu}{\mu+v}} \mathbb{E}_{\mu+v, v+1}(\lambda)\right]\|g\|_{\infty} .
\end{aligned}
$$

From the above the proof is complete.

Lemma 2.4 For $\lambda>0$, define

$$
\begin{aligned}
Q(\mu, v, \lambda)= & \max \left\{\mathbb{E}_{\mu+v, \mu+v+1}(-\lambda)+\frac{m(\mu+v, \mu+v, \lambda)}{\mu+v},\right. \\
& \frac{1}{(\mu+v) \lambda}+2 \lambda^{\frac{-\mu}{\mu+v}} m(\mu+v, v+1, \lambda), \\
& \left.\frac{m(\mu+v, \mu+v, \lambda)}{\mu+v}+\mathbb{E}_{\mu+v, \mu+v+1}(\lambda)+4 \lambda^{\frac{-\mu}{\mu+\nu}} m(\mu+v, v+1, \lambda)\right\} .
\end{aligned}
$$

Then, for any function $g \in X_{\infty}\left(\mathbb{R}_{+}, \mathbb{R}\right)$, the following statements hold for all $t>1$ :

(i) $\quad\left|\int_{0}^{t}(t-s)^{\mu+\nu-1} \mathbb{E}_{\mu+v, \mu+v}\left(-(t-s)^{\mu+v} \lambda\right) g(s) d s\right| \leq Q(\mu, v, \lambda)\|g\|_{\infty}$.

(ii) $\left|\lambda^{\frac{1-\mu}{\mu+\nu}} t^{\nu} \mathbb{E}_{\mu+v, v+1}\left(t^{\mu+v} \lambda\right) \int_{t}^{\infty} \exp \left(-\lambda^{\frac{1}{\mu+v}} s\right) g(s) d s\right| \leq Q(\mu, v, \lambda)\|g\|_{\infty}$.

(iii) $\mid \int_{0}^{t}\left[(t-s)^{\mu+v-1} \mathbb{E}_{\mu+v, \mu+v}\left((t-s)^{\mu+v} \lambda\right)\right.$

$$
\begin{aligned}
& \left.-\lambda^{\frac{1-\mu}{\mu+\nu}} t^{v} \mathbb{E}_{\mu+v, v+1}\left(t^{\mu+v} \lambda\right) \exp \left(-\lambda^{\frac{1}{\mu+\nu}} s\right)\right] g(s) d s \mid \\
\leq & Q(\mu, \nu, \lambda)\|g\|_{\infty} .
\end{aligned}
$$

Proof

(i) Note that

$$
\int_{t-1}^{t}(t-s)^{\mu+\nu-1} \mathbb{E}_{\mu+v, \mu+\nu}\left(-(t-s)^{\mu+v} \lambda\right) d s=\mathbb{E}_{\mu+v, \mu+v+1}(-\lambda),
$$

So

$$
\left|\int_{t-1}^{t}(t-s)^{\mu+v-1} \mathbb{E}_{\mu+v, \mu+v}\left(-(t-s)^{\mu+v} \lambda\right) g(s) d s\right| \leq \mathbb{E}_{\mu+v, \mu+v+1}(-\lambda)\|g\|_{\infty} .
$$


Wang et al. Advances in Difference Equations (2017) 2017:335

Page 6 of 15

On the other hand, applying Lemma 2.1(i), we get

$$
\begin{aligned}
& \left|\int_{0}^{t-1}(t-s)^{\mu+\nu-1} \mathbb{E}_{\mu+v, \mu+\nu}\left(-(t-s)^{\mu+\nu} \lambda\right) g(s) d s\right| \\
& \quad \leq \int_{0}^{t-1}\left|(t-s)^{\mu+\nu-1} \mathbb{E}_{\mu+\nu, \mu+\nu}\left(-(t-s)^{\mu+v} \lambda\right)\right||g(s)| d s \\
& \quad \leq \int_{0}^{t-1} \frac{m(\mu+v, \mu+v, \lambda)}{(t-s)^{1+\mu+v}}|g(s)| d s \\
& \quad \leq \frac{m(\mu+v, \mu+v, \lambda)}{\mu+v}\|g\|_{\infty} .
\end{aligned}
$$

Consequently, we get

$$
\begin{aligned}
& \left|\int_{0}^{t}(t-s)^{\mu+\nu-1} \mathbb{E}_{\mu+v, \mu+v}\left(-(t-s)^{\mu+v} \lambda\right) g(s) d s\right| \\
& \quad \leq\left(\mathbb{E}_{\mu+v, \mu+v+1}(-\lambda)+\frac{m(\mu+\nu, \mu+v, \lambda)}{\mu+v}\right)\|g\|_{\infty} .
\end{aligned}
$$

(ii) Similarly, applying Lemma 2.1(ii), we get

$$
\begin{aligned}
& \left|\lambda^{\frac{1-\mu}{\mu+v}} t^{\nu} \mathbb{E}_{\mu+\nu, v+1}\left(t^{\mu+v} \lambda\right) \int_{t}^{\infty} \exp \left(-\lambda^{\frac{1}{\mu+\nu}} s\right) g(s) d s\right| \\
& \leq \lambda^{\frac{1-\mu}{\mu+\nu}}\left[m(\mu+\nu, v+1, \lambda)\left(\frac{1}{t^{2 \mu+\nu}}+\frac{1}{t^{\mu}}\right)\right. \\
& \left.+\frac{1}{\mu+v} \lambda^{\frac{-v}{\mu+\nu}} \exp \left(\lambda^{\frac{1}{\mu+\nu}} t\right)\right] \int_{t}^{\infty} \exp \left(-\lambda^{\frac{1}{\mu+\nu}} s\right)|g(s)| d s \\
& \leq \frac{1}{\mu+v} \lambda^{\frac{1-\mu-v}{\mu+\nu}} \int_{t}^{\infty} \exp \left(\lambda^{\frac{1}{\mu+v}}(t-s)\right)|g(s)| d s \\
& +2 \lambda^{\frac{1-\mu}{\mu+\nu}} m(\mu+v, v+1, \lambda) \int_{t}^{\infty} \exp \left(-\lambda^{\frac{1}{\mu+v}} s\right)|g(s)| d s \\
& \leq\left(\frac{1}{(\mu+v) \lambda}+2 \lambda^{\frac{-\mu}{\mu+v}} m(\mu+v, v+1, \lambda)\right)\|g\|_{\infty} .
\end{aligned}
$$

(iii) Like the above we get

$$
\begin{aligned}
& \left|(t-s)^{\mu+v-1} \mathbb{E}_{\mu+v, \mu+\nu}\left((t-s)^{\mu+v} \lambda\right)-\lambda^{\frac{1-\mu}{\mu+\nu}} t^{\nu} \mathbb{E}_{\mu+v, v+1}\left(t^{\mu+v} \lambda\right) \exp \left(-\lambda^{\frac{1}{\mu+\nu}} s\right)\right| \\
& \quad \leq \frac{m(\mu+v, \mu+v, \lambda)}{(t-s)^{\mu+\nu+1}}+m(\mu+v, v+1, \lambda) \lambda^{\frac{1-\mu}{\mu+\nu}} \exp \left(-\lambda^{\frac{1}{\mu+\nu}} s\right)\left(\frac{1}{t^{2 \mu+\nu}}+\frac{1}{t^{\mu}}\right),
\end{aligned}
$$

SO

$$
\begin{aligned}
& \mid \int_{0}^{t-1}\left[(t-s)^{\mu+\nu-1} \mathbb{E}_{\mu+v, \mu+v}\left((t-s)^{\mu+v} \lambda\right)\right. \\
& \left.\quad-\lambda^{\frac{1-\mu}{\mu+\nu}} t^{\nu} \mathbb{E}_{\mu+v, v+1}\left(t^{\mu+v} \lambda\right) \exp \left(-\lambda^{\frac{1}{\mu+v}} s\right)\right] g(s) d s \mid \\
& \quad \leq \int_{0}^{t-1} \frac{m(\mu+v, \mu+v, \lambda)|g(s)|}{(t-s)^{\mu+v+1}} d s
\end{aligned}
$$




$$
\begin{aligned}
& +2 m(\mu+v, v+1, \lambda) \lambda^{\frac{1-\mu}{\mu+\nu}} \int_{0}^{t-1} \exp \left(-\lambda^{\frac{1}{\mu+\nu}} s\right) d s\|g\|_{\infty} \\
\leq & {\left[\frac{m(\mu+v, \mu+v, \lambda)}{\mu+v}+2 m(\mu+v, v+1, \lambda) \lambda^{\frac{-\mu}{\mu+\nu}}\right]\|g\|_{\infty} . }
\end{aligned}
$$

On the other hand,

$$
\begin{aligned}
\mid \int_{t-1}^{t} & {\left[(t-s)^{\mu+\nu-1} \mathbb{E}_{\mu+\nu, \mu+\nu}\left((t-s)^{\mu+\nu} \lambda\right)\right.} \\
& \left.-\lambda^{\frac{1-\mu}{\mu+\nu}} t^{\nu} \mathbb{E}_{\mu+\nu, v+1}\left(t^{\mu+\nu} \lambda\right) \exp \left(-\lambda^{\frac{1}{\mu+\nu}} s\right)\right] g(s) d s \mid \\
\leq & {\left[\mathbb{E}_{\mu+\nu, \mu+\nu+1}(\lambda)+\int_{t-1}^{t} \lambda^{\frac{1-\mu}{\mu+\nu}} t^{\nu} \mathbb{E}_{\mu+\nu, v+1}\left(t^{\mu+\nu} \lambda\right) \exp \left(-\lambda^{\frac{1}{\mu+\nu}} s\right) d s\right]\|g\|_{\infty} . }
\end{aligned}
$$

Furthermore, we obtain

$$
\begin{aligned}
& \left|\int_{t-1}^{t} \lambda^{\frac{1-\mu}{\mu+\nu}} t^{v} \mathbb{E}_{\mu+v, v+1}\left(t^{\mu+v} \lambda\right) \exp \left(-\lambda^{\frac{1}{\mu+\nu}} s\right) d s\right| \\
& \quad \leq \lambda^{\frac{1-\mu}{\mu+\nu}} m(\mu+v, v+1, \lambda)\left(\frac{1}{t^{2 \mu+v}}+\frac{1}{t^{\mu}}\right) \int_{t-1}^{t} \exp \left(-\lambda^{\frac{1}{\mu+\nu}} s\right) d s \\
& \quad \leq 2 \lambda^{\frac{-\mu}{\mu+\nu}} m(\mu+v, v+1, \lambda) .
\end{aligned}
$$

Consequently,

$$
\begin{aligned}
& \mid \int_{0}^{t}\left[(t-s)^{\mu+\nu-1} \mathbb{E}_{\mu+\nu, \mu+\nu}\left((t-s)^{\mu+\nu} \lambda\right)\right. \\
& \left.-\lambda^{\frac{1-\mu}{\mu+\nu}} t^{v} \mathbb{E}_{\mu+v, v+1}\left(t^{\mu+v} \lambda\right) \exp \left(-\lambda^{\frac{1}{\mu+\nu}} s\right)\right] g(s) d s \mid \\
& \leq\left[\frac{m(\mu+v, \mu+v, \lambda)}{\mu+v}+\mathbb{E}_{\mu+v, \mu+v+1}(\lambda)+4 \lambda^{\frac{-\mu}{\mu+v}} m(\mu+v, v+1, \lambda)\right]\|g\|_{\infty} .
\end{aligned}
$$

From the above the proof is complete.

\section{Local center stable manifold theorem}

From Lemmas 2.3 and 2.4 , the operator $\mathcal{L P}$ in (4) is well defined. We now state and prove some fundamental properties of $\mathcal{L P}$, which are used later to prove the existence of stable manifolds.

\section{Proposition 3.1 Define}

$$
B\left(\mu, \nu, \lambda_{1}, \lambda_{2}\right)=\max \left\{P\left(\mu, v, \lambda_{1}\right), Q\left(\mu, v, \lambda_{1}\right), 2 P\left(\mu, \nu,-\lambda_{2}\right), 2 Q\left(\mu, v,-\lambda_{2}\right)\right\},
$$

where $P$ and $Q$ are the functions defined as in Lemmas 2.3 and 2.4. For any $\eta, \hat{\eta} \in$ $X_{\infty}\left(\mathbb{R}_{+}, \mathbb{R}^{2}\right)$, we have

$$
\|\mathcal{L} \mathcal{P}(\eta)-\mathcal{L} \mathcal{P}(\hat{\eta})\|_{\infty} \leq B\left(\mu, \nu, \lambda_{1}, \lambda_{2}\right) l_{h}\left(\max \left(\|\eta\|_{\infty},\|\hat{\eta}\|_{\infty}\right)\right)\|\eta-\hat{\eta}\|_{\infty}
$$


and

$$
\begin{aligned}
\|\mathcal{L} \mathcal{P}(\eta)\|_{\infty} \leq & \left(\frac{1}{\Gamma(v+1)}+2 m\left(\mu+v, v+1, \lambda_{1}\right)\right)\left|x_{3}\right| \\
& +B\left(\mu, v, \lambda_{1}, \lambda_{2}\right) l_{h}\left(\|\eta\|_{\infty}\right)\|\eta\|_{\infty} .
\end{aligned}
$$

Proof Note that

$$
\begin{aligned}
& \left|\mathcal{L P}_{1}(\eta)-\mathcal{L P}_{1}(\hat{\eta})\right| \\
& \quad \leq\left|\int_{0}^{t}(t-s)^{\mu+\nu-1} \mathbb{E}_{\mu+\nu, \mu+v}\left(-(t-s)^{\mu+\nu} \lambda_{1}\right)\left(h_{1}(\eta(s), s)-h_{1}(\hat{\eta}(s), s)\right) d s\right| .
\end{aligned}
$$

Now using Lemmas 2.3 and 2.4, we have

$$
\sup _{t \in[0,1]}\left|\mathcal{L} \mathcal{P}_{1}(\eta)(t)-\mathcal{L} \mathcal{P}_{1}(\hat{\eta})(t)\right| \leq P\left(\mu, v, \lambda_{1}\right) l_{h}\left(\max \left(\|\eta\|_{\infty},\|\hat{\eta}\|_{\infty}\right)\right)\|\eta-\hat{\eta}\|_{\infty}
$$

and

$$
\sup _{t>1}\left|\mathcal{L} \mathcal{P}_{1}(\eta)(t)-\mathcal{L} \mathcal{P}_{1}(\hat{\eta})(t)\right| \leq Q\left(\mu, v, \lambda_{1}\right) l_{h}\left(\max \left(\|\eta\|_{\infty},\|\hat{\eta}\|_{\infty}\right)\right)\|\eta-\hat{\eta}\|_{\infty}
$$

so

$$
\sup _{t \geq 0}\left|\mathcal{L} \mathcal{P}_{1}(\eta)(t)-\mathcal{L} \mathcal{P}_{1}(\hat{\eta})(t)\right| \leq B\left(\mu, v, \lambda_{1}, \lambda_{2}\right) l_{h}\left(\max \left(\|\eta\|_{\infty},\|\hat{\eta}\|_{\infty}\right)\right)\|\eta-\hat{\eta}\|_{\infty}
$$

On the other hand,

$$
\begin{aligned}
\left|\mathcal{L P}_{2}(\eta)(t)-\mathcal{L P}_{2}(\hat{\eta})(t)\right| \\
=\mid-\left(-\lambda_{2}\right)^{\frac{1-\mu}{\mu+v}} t^{v} \mathbb{E}_{\mu+v, v+1}\left(-\lambda_{2} t^{\mu+v}\right) \int_{0}^{\infty} \exp \left(-\left(-\lambda_{2}\right)^{\frac{1}{\mu+\nu}} s\right)\left(h_{2}(\eta(s), s)-h_{2}(\hat{\eta}(s), s)\right) d s \\
\quad+\int_{0}^{t}(t-s)^{\mu+v-1} \mathbb{E}_{\mu+v, \mu+v}\left(-(t-s)^{\mu+v} \lambda_{2}\right)\left(h_{2}(\eta(s), s)-h_{2}(\hat{\eta}(s), s)\right) d s \mid \\
=\mid \int_{0}^{t}\left((t-s)^{\mu+v-1} \mathbb{E}_{\mu+v, \mu+v}\left(-(t-s)^{\mu+v} \lambda_{2}\right)\right. \\
\left.\quad-\left(-\lambda_{2}\right)^{\frac{1-\mu}{\mu+v}} t^{v} \mathbb{E}_{\mu+v, v+1}\left(-\lambda_{2} t^{\mu+v}\right) \exp \left(-\left(-\lambda_{2}\right)^{\frac{1}{\mu+\nu}} s\right)\right)\left(h_{2}(\eta(s), s)-h_{2}(\hat{\eta}(s), s)\right) d s \\
\quad-\left(-\lambda_{2}\right)^{\frac{1-\mu}{\mu+\nu}} t^{\nu} \mathbb{E}_{\mu+v, v+1}\left(-\lambda_{2} t^{\mu+v}\right) \\
\quad \times \int_{t}^{\infty} \exp \left(-\left(-\lambda_{2}\right)^{\frac{1}{\mu+v}} s\right)\left(h_{2}(\eta(s), s)-h_{2}(\hat{\eta}(s), s)\right) d s \mid .
\end{aligned}
$$

From Lemmas 2.3 and 2.4, we have

$$
\sup _{t \in[0,1]}\left|\mathcal{L} \mathcal{P}_{2}(\eta)(t)-\mathcal{L} \mathcal{P}_{2}(\hat{\eta})(t)\right| \leq 2 P\left(\mu, \nu,-\lambda_{2}\right) l_{h}\left(\max \left(\|\eta\|_{\infty},\|\hat{\eta}\|_{\infty}\right)\right)\|\eta-\hat{\eta}\|_{\infty}
$$

and

$$
\sup _{t>1}\left|\mathcal{L} \mathcal{P}_{2}(\eta)(t)-\mathcal{L} \mathcal{P}_{2}(\hat{\eta})(t)\right| \leq 2 Q\left(\mu, v,-\lambda_{2}\right) l_{h}\left(\max \left(\|\eta\|_{\infty},\|\hat{\eta}\|_{\infty}\right)\right)\|\eta-\hat{\eta}\|_{\infty}
$$


so

$$
\sup _{t \geq 0}\left|\mathcal{L} \mathcal{P}_{2}(\eta)(t)-\mathcal{L} \mathcal{P}_{2}(\hat{\eta})(t)\right| \leq B\left(\mu, v, \lambda_{1}, \lambda_{2}\right) l_{h}\left(\max \left(\|\eta\|_{\infty},\|\hat{\eta}\|_{\infty}\right)\right)\|\eta-\hat{\eta}\|_{\infty}
$$

Consequently, we get conclusion (6). Next, notice

$$
\begin{aligned}
& \left|\mathcal{L P}_{1}(0)(t)\right| \leq\left|t^{v} \mathbb{E}_{\mu+v, v+1}\left(-t^{\mu+v} \lambda_{1}\right)\right|\left|x_{3}\right| \leq\left(\frac{1}{\Gamma(v+1)}+2 m\left(\mu+v, v+1, \lambda_{1}\right)\right)\left|x_{3}\right|, \\
& \left|\mathcal{L} \mathcal{P}_{2}(0)(t)\right|=0
\end{aligned}
$$

Hence we get conclusion (7). The proof is complete.

Before stating and proving the stable invariant manifold result, we present the following technical lemma.

Lemma 3.2 For any function $g \in X_{\infty}\left(\mathbb{R}_{+}, \mathbb{R}\right)$ and $\lambda>0$, we have

$$
\lim _{u \rightarrow \infty} \frac{\int_{0}^{u}(u-s)^{\mu+\nu-1} \mathbb{E}_{\mu+v, \mu+\nu}\left((u-s)^{\mu+v} \lambda\right) g(s) d s}{u^{\nu} \mathbb{E}_{\mu+v, v+1}\left(\lambda u^{\mu+v}\right)}=\lambda^{\frac{1-\mu}{\mu+\nu}} \int_{0}^{\infty} \exp \left(-\lambda^{\frac{1}{\mu+\nu}} s\right) g(s) d s .
$$

Proof According to Lemma 2.1(ii), we obtain

$$
\lim _{u \rightarrow \infty} \frac{\frac{1}{\mu+\nu} \lambda^{\frac{-v}{\mu+v}} \exp \left(\lambda^{\frac{1}{\mu+v}} u\right)}{u^{v} \mathbb{E}_{\mu+v, v+1}\left(\lambda u^{\mu+v}\right)}=1
$$

Next, since for $u>1$

$$
\left|\int_{u-1}^{u}(u-s)^{\mu+v-1} \mathbb{E}_{\mu+v, \mu+v}\left((u-s)^{\mu+v} \lambda\right) g(s) d s\right| \leq \mathbb{E}_{\mu+v, \mu+v+1}(\lambda)\|g\|_{\infty},
$$

then

$$
\lim _{u \rightarrow \infty} \int_{u-1}^{u} \frac{(u-s)^{\mu+\nu-1} \mathbb{E}_{\mu+\nu, \mu+\nu}\left((u-s)^{\mu+\nu} \lambda\right)}{\frac{1}{\mu+\nu} \lambda^{\frac{-v}{\mu+\nu}} \exp \left(\lambda^{\frac{1}{\mu+\nu}} u\right)} g(s) d s=0 .
$$

Also we have

$$
\begin{aligned}
& \lim _{u \rightarrow \infty}\left|\int_{0}^{u-1} \frac{(u-s)^{\mu+\nu-1} \mathbb{E}_{\mu+v, \mu+v}\left((u-s)^{\mu+v} \lambda\right)-\frac{1}{\mu+\nu} \lambda^{\frac{1-\mu-v}{\mu+\nu}} \exp \left(\lambda^{\frac{1}{\mu+v}}(u-s)\right)}{\frac{1}{\mu+\nu} \lambda^{\frac{-v}{\mu+\nu}} \exp \left(\lambda^{\frac{1}{\mu+v}} u\right)} g(s) d s\right| \\
& \quad \leq \lim _{u \rightarrow \infty} \int_{0}^{u-1} \frac{m(\mu+v, \mu+v, \lambda)}{\frac{1}{\mu+\nu} \lambda^{\frac{-v}{\mu+\nu}} \exp \left(\lambda^{\frac{1}{\mu+\nu}} u\right)(u-s)^{1+\mu+v}} d s\|g\|_{\infty}=0,
\end{aligned}
$$

so

$$
\begin{aligned}
& \lim _{u \rightarrow \infty} \int_{0}^{u} \frac{(u-s)^{\mu+\nu-1} \mathbb{E}_{\mu+v, \mu+v}\left((u-s)^{\mu+v} \lambda\right)}{\frac{1}{\mu+\nu} \lambda^{\frac{-v}{\mu+\nu}} \exp \left(\lambda^{\frac{1}{\mu+\nu}} u\right)} g(s) d s \\
& \quad=\lim _{u \rightarrow \infty} \int_{0}^{u} \frac{\lambda^{\frac{1-\mu}{\mu+\nu}} \exp \left(\lambda^{\frac{1}{\mu+\nu}}(u-s)\right)}{\exp \left(\lambda^{\frac{1}{\mu+\nu}} u\right)} g(s) d s=\lambda^{\frac{1-\mu}{\mu+\nu}} \int_{0}^{\infty} \exp \left(-\lambda^{\frac{1}{\mu+v}} s\right) g(s) d s .
\end{aligned}
$$

The proof is complete. 
Let $V \subset U \subset \mathbb{R}^{2}$ and $W \subset \mathbb{R}^{2}$ be open neighborhoods of $\mathbf{0}$. Define a local center stable manifold

$$
W_{0}^{c s}(V \times W, U)=\{\mathbf{0} \in V, \bar{x} \in W: \phi(t, \mathbf{0}, \bar{x}) \in U, \forall t \geq 0\} .
$$

Proposition $3.3(\mathbf{0}, \bar{x}) \in W_{0}^{c s}(V \times W, U)$ if and only if $\phi(\cdot, \mathbf{0}, \bar{x})$ is a fixed point of $\mathcal{L P}$ along with $\phi(t, \mathbf{0}, \bar{x}) \in U \forall t \geq 0$. Furthermore, $\lim _{t \rightarrow \infty} \phi(t, \mathbf{0}, \bar{x})=0$ provided that $\rho=$ $l_{h}\left(r^{*}\right) B\left(\mu, v, \lambda_{1}, \lambda_{2}\right)<1$ for $\|\phi\|_{\infty}<r^{*}$, where $B\left(\mu, v, \lambda_{1}, \lambda_{2}\right)$ is defined in Proposition 3.1.

Proof If $(\mathbf{0}, \bar{x}) \in W_{0}^{c s}(V \times W, U)$, then from (3) we get

$$
\begin{aligned}
\phi_{1}(t, \mathbf{0}, \bar{x})= & t^{\nu} \mathbb{E}_{\mu+v, v+1}\left(-t^{\mu+v} \lambda_{1}\right) x_{3} \\
& +\int_{0}^{t}(t-s)^{\mu+\nu-1} \mathbb{E}_{\mu+v, \mu+v}\left(-(t-s)^{\mu+\nu} \lambda_{1}\right) h_{1}(\phi(s, \mathbf{0}, \bar{x}), s) d s,
\end{aligned}
$$

and

$$
\begin{aligned}
\phi_{2}(t, \mathbf{0}, \bar{x})= & t^{\nu} \mathbb{E}_{\mu+v, v+1}\left(-t^{\mu+v} \lambda_{2}\right) x_{4} \\
& +\int_{0}^{t}(t-s)^{\mu+\nu-1} \mathbb{E}_{\mu+v, \mu+v}\left(-(t-s)^{\mu+\nu} \lambda_{2}\right) h_{2}(\phi(s, \mathbf{0}, \bar{x}), s) d s .
\end{aligned}
$$

From the above results we know that $\phi_{1}(\cdot, x, \bar{x})=\mathcal{L P}_{1}(\phi(\cdot, x, \bar{x}))$.

Furthermore, $\lim _{t \rightarrow \infty} t^{v} \mathbb{E}_{\mu+v, v+1}\left(-t^{\mu+v} \lambda_{2}\right)=\infty$ and using $\phi_{2} \in X_{\infty}\left(\mathbb{R}_{+}, \mathbb{R}\right)$, we arrive at

$$
\begin{aligned}
x_{4} & =-\lim _{t \rightarrow \infty} \frac{\int_{0}^{t}(t-s)^{\mu+\nu-1} \mathbb{E}_{\mu+v, \mu+\nu}\left(-(t-s)^{\mu+v} \lambda_{2}\right) h_{2}(\phi(s, \mathbf{0}, \bar{x}), s) d s}{t^{v} \mathbb{E}_{\mu+v, v+1}\left(-t^{\mu+\nu} \lambda_{2}\right)} \\
& =-\left(-\lambda_{2}\right)^{\frac{1-\mu}{\mu+\nu}} \int_{0}^{\infty} \exp \left(-\left(-\lambda_{2}\right)^{\frac{1}{\mu+\nu}} s\right) h_{2}(\phi(s, \mathbf{0}, \bar{x}), s) d s,
\end{aligned}
$$

because of Lemma 3.2. Hence

$$
\begin{aligned}
\phi_{2}(t, \mathbf{0}, \bar{x})= & -\left(-\lambda_{2}\right)^{\frac{1-\mu}{\mu+\nu}} t^{\nu} \mathbb{E}_{\mu+\nu, v+1}\left(-t^{\mu+\nu} \lambda_{2}\right) \int_{0}^{\infty} \exp \left(-\left(-\lambda_{2}\right)^{\frac{1}{\mu+\nu}} s\right) h_{2}(\phi(s, \mathbf{0}, \bar{x}), s) d s \\
& +\int_{0}^{t}(t-s)^{\mu+\nu-1} \mathbb{E}_{\mu+\nu, \mu+\nu}\left(-(t-s)^{\mu+\nu} \lambda_{2}\right) h_{2}(\phi(s, \mathbf{0}, \bar{x}), s) d s=\mathcal{T}_{2}\left(\phi_{2}(\cdot, \mathbf{0}, \bar{x})\right),
\end{aligned}
$$

so $\phi(t, \mathbf{0}, \bar{x})$ is a fixed point of $\mathcal{L P}$. Clearly, $\phi(t, \mathbf{0}, \bar{x}) \in U, \forall t \geq 0$.

On the other hand, let $\eta \in X_{\infty}\left(\mathbb{R}_{+}, \mathbb{R}^{2}\right) \cap X^{1}\left(\mathbb{R}_{+}, \mathbb{R}^{2}\right),\left(\eta(0), \eta^{\prime}(0)\right) \in V \times W$ be a fixed point of $\mathcal{T}$ such that $\eta(t) \in U, \forall t \geq 0$. Then

$$
\begin{aligned}
\eta_{1}(t)= & t^{\nu} \mathbb{E}_{\mu+v, v+1}\left(-t^{\mu+v} \lambda_{1}\right) x_{3}+\int_{0}^{t}(t-s)^{\mu+\nu-1} \mathbb{E}_{\mu+v, \mu+v}\left(-(t-s)^{\mu+v} \lambda_{1}\right) h_{1}(\eta(s), s) d s, \\
\eta_{2}(t)= & -\left(-\lambda_{2}\right)^{\frac{1-\mu}{\mu+\nu}} t^{\nu} \mathbb{E}_{\mu+v, v+1}\left(-t^{\mu+v} \lambda_{2}\right) \int_{0}^{\infty} \exp \left(-\left(-\lambda_{2}\right)^{\frac{1}{\mu+\nu}} s\right) h_{2}(\eta(s), s) d s \\
& +\int_{0}^{t}(t-s)^{\mu+\nu-1} \mathbb{E}_{\mu+v, \mu+\nu}\left(-(t-s)^{\mu+\nu} \lambda_{2}\right) h_{2}(\eta(s), s) d s .
\end{aligned}
$$


Defining

$$
x_{4}=-\left(-\lambda_{2}\right)^{\frac{1-\mu}{\mu+\nu}} \int_{0}^{\infty} \exp \left(-\left(-\lambda_{2}\right)^{\frac{1}{\mu+\nu}} s\right) h_{2}(\eta(s), s) d s
$$

we get

$$
\begin{aligned}
\eta(t)= & \phi(t, \mathbf{0}, \bar{x}) \\
= & t^{\nu} \mathbb{E}_{\mu+v, v+1}\left(-t^{\mu+v} A\right) \bar{x} \\
& +\int_{0}^{t}(t-s)^{\mu+\nu-1} \mathbb{E}_{\mu+v, \mu+v}\left(-(t-s)^{\mu+v} A\right) h(\phi(s, \mathbf{0}, \bar{x})) d s,
\end{aligned}
$$

with $\eta(0)=(0,0)^{T}$ and $\eta^{\prime}(0)=\left(x_{3}, x_{4}\right)^{T}$, which is a bounded solution of (1).

Finally, we check that $\lim _{t \rightarrow \infty} \sup \|\eta(t)\|=0$. Let $\lim _{t \rightarrow \infty} \sup \|\eta(t)\|=a \in\left[0, r^{*}\right)$. Thus, there exists a sequence $\left\{t_{n}\right\}$ such that $\lim _{n \rightarrow \infty} t_{n}=\infty$ with $\lim _{n \rightarrow \infty} \sup \left\|\eta\left(t_{n}\right)\right\|=a$. That is, $\forall \epsilon>0, \exists T(\epsilon)>0$, when $t>T(\epsilon)$, we have $\|\eta(t)\|<a+\epsilon:=r^{*}$. On the one hand, it follows (5) that

$$
\left|\int_{T(\epsilon)}^{t}(t-s)^{\mu+\nu-1} \mathbb{E}_{\mu+\nu, \mu+v}\left(-(t-s)^{\mu+v} \lambda_{1}\right) d s\right| \leq \int_{0}^{T(\epsilon)} \frac{m\left(\mu+v, \mu+v, \lambda_{1}\right)}{(t-s)^{1+\mu+\nu}} d s .
$$

On the other hand, repeat a proof similar to that in Lemma 2.3(i), Lemma 2.4(i), and one can derive that

$$
\left|\int_{T(\epsilon)}^{t}(t-s)^{\mu+v-1} \mathbb{E}_{\mu+v, \mu+v}\left(-(t-s)^{\mu+v} \lambda_{1}\right) d s\right| \leq B\left(\mu, \nu, \lambda_{1}, \lambda_{2}\right)
$$

From Lemma 2.1(i), (8) and (9), and the fact that $h$ is a Lipschitz type function with $h(0, t)=0$, one obtains

$$
\begin{aligned}
\lim _{t \rightarrow \infty} \sup \left|\eta_{1}(t)\right|= & \lim _{t \rightarrow \infty} \sup \left[\left|t^{\nu} \mathbb{E}_{\mu+\nu, v+1}\left(-t^{\mu+\nu} \lambda_{1}\right)\right|\left|x_{3}\right|\right. \\
& +\left|\int_{0}^{T(\epsilon)}(t-s)^{\mu+\nu-1} \mathbb{E}_{\mu+\nu, \mu+\nu}\left(-(t-s)^{\mu+\nu} \lambda_{1}\right) h_{1}(\eta(s), s) d s\right| \\
& \left.+\left|\int_{T(\epsilon)}^{t}(t-s)^{\mu+\nu-1} \mathbb{E}_{\mu+\nu, \mu+\nu}\left(-(t-s)^{\mu+\nu} \lambda_{1}\right) h_{1}(\eta(s)) d s\right|\right] \\
\leq & \lim _{t \rightarrow \infty} \sup \left[m\left(\mu+v, v+1, \lambda_{1}\right)\left(\frac{1}{t^{2 \mu+\nu}}+\frac{1}{t^{\mu}}\right)\left|x_{3}\right|\right. \\
& +r^{*} l_{h}\left(r^{*}\right) \int_{0}^{T(\epsilon)} \frac{m\left(\mu+v, \mu+v, \lambda_{1}\right)}{(t-s)^{1+\mu+v}} d s \\
& \left.+r^{*} l_{h}\left(r^{*}\right)\left|\int_{T(\epsilon)}^{t}(t-s)^{\mu+\nu-1} \mathbb{E}_{\mu+\nu, \mu+\nu}\left(-(t-s)^{\mu+\nu} \lambda_{1}\right) d s\right|\right] \\
= & r^{*} l_{h}\left(r^{*}\right) B\left(\mu, v, \lambda_{1}, \lambda_{2}\right)=\rho r^{*} .
\end{aligned}
$$

Thus $\lim _{t \rightarrow \infty} \sup \left|\eta_{1}(t)\right| \leq \rho r^{*}=\rho(a+\epsilon)$. 
Also, one can use Lemma 3.2, so

$$
\begin{aligned}
& \lim _{t \rightarrow \infty} \sup \left|\eta_{2}(t)\right| \\
& =\lim _{t \rightarrow \infty} \sup \mid-\left(-\lambda_{2}\right)^{\frac{1-\mu}{\mu+v}} t^{\nu} \mathbb{E}_{\mu+v, v+1}\left(-t^{\mu+v} \lambda_{2}\right) \int_{0}^{\infty} \exp \left(-\left(-\lambda_{2}\right)^{\frac{1}{\mu+v}} s\right) h_{2}(\eta(s), s) d s \\
& +\int_{0}^{t}(t-s)^{\mu+\nu-1} \mathbb{E}_{\mu+\nu, \mu+\nu}\left(-(t-s)^{\mu+\nu} \lambda_{2}\right) h_{2}(\eta(s), s) d s \mid \\
& =\lim _{t \rightarrow \infty} \sup \mid t^{\nu} \mathbb{E}_{\mu+\nu, v+1}\left(-t^{\mu+\nu} \lambda_{2}\right)\left[-\left(-\lambda_{2}\right)^{\frac{1-\mu}{\mu+\nu}} \int_{0}^{\infty} \exp \left(-\left(-\lambda_{2}\right)^{\frac{1}{\mu+\nu}} s\right) h_{2}(\eta(s), s) d s\right. \\
& \left.+\frac{\int_{0}^{t}(t-s)^{\mu+\nu-1} \mathbb{E}_{\mu+v, \mu+v}\left(-(t-s)^{\mu+\nu} \lambda_{2}\right) h_{2}(\eta(s), s) d s}{t^{v} \mathbb{E}_{\mu+\nu, v+1}\left(-t^{\mu+v} \lambda_{2}\right)}\right] \mid \\
& =0 \text {. }
\end{aligned}
$$

From the above,

$$
\lim _{t \rightarrow \infty} \sup \|\eta(t)\|=\max \left(\lim _{t \rightarrow \infty} \sup \left|\eta_{1}(t)\right|, \lim _{t \rightarrow \infty} \sup \left|\eta_{2}(t)\right|\right)=a
$$

Thus, we get $a \leq \rho(a+\epsilon)$, which yields that $(1-\rho) a \leq 0$. Letting $\epsilon \rightarrow 0$, we obtain $a=0$ since $\rho<1$. The proof is complete.

Now we state and prove the main result on stable manifolds.

Theorem 3.4 Take $\vartheta^{*}>0$ and set

$$
\begin{aligned}
& \vartheta=\frac{(1-\rho) \vartheta^{*}}{\frac{1}{\Gamma(\nu+1)}+2 m\left(\mu+\nu, v+1, \lambda_{1}\right)}, \\
& \vartheta^{* *}=\left(-\lambda_{2}\right)^{\frac{-\mu}{\mu+v}} l_{h}\left(\vartheta^{*}\right) \vartheta^{*} .
\end{aligned}
$$

Then, for any $\tilde{x}=\left(0,0, x_{3}\right) \in(-\vartheta, \vartheta)^{3}$, there exists a unique $\mathcal{S}(\tilde{x}) \in\left(-\vartheta^{* *}, \vartheta^{* *}\right)$ such that $(\tilde{x}, \mathcal{S}(\tilde{x})) \in W_{0}^{c s}(V \times W, U)$ with $V=(-\vartheta, \vartheta)^{2}, W=(-\vartheta, \vartheta) \times\left(-\vartheta^{* *}, \vartheta^{* *}\right)$ and $U=$ $\left(-\vartheta^{*}, \vartheta^{*}\right)^{2}$. Furthermore, $\mathcal{S}:(-\vartheta, \vartheta)^{3} \rightarrow\left(-\vartheta^{* *}, \vartheta^{* *}\right)$ has the following properties:

(i) $\mathcal{S}(0)=0$.

(ii) $\mathcal{S}$ is Lipschitz continuous:

$$
|\mathcal{S}(\tilde{x})-\mathcal{S}(\tilde{y})| \leq \frac{\vartheta^{* *}}{\vartheta^{*}(1-\rho)}\left(\frac{1}{\Gamma(v+1)}+2 m\left(\mu+v, v+1, \lambda_{1}\right)\right)\left|x_{3}-y_{3}\right|
$$

for any $\tilde{x}=\left(x, x_{3}\right), \tilde{y}=\left(y, y_{3}\right) \in(-\vartheta, \vartheta)^{3}$.

Proof Let $\mathbf{B}_{\vartheta^{*}}(0)=\left\{\eta \in X_{\infty}\left(\mathbb{R}_{+}, \mathbb{R}^{2}\right):\|\eta\|_{\infty} \leq \vartheta^{*}\right\}$. From Proposition 3.1, we have

$$
\begin{aligned}
& \|\mathcal{L P}(\eta)-\mathcal{L P}(\hat{\eta})\|_{\infty} \leq B\left(\mu, \nu, \lambda_{1}, \lambda_{2}\right) l_{h}\left(\vartheta^{*}\right)\|\eta-\hat{\eta}\|_{\infty}=\rho\|\eta-\hat{\eta}\|_{\infty} \\
& \|\mathcal{L P}(\eta)\|_{\infty} \leq\left(\frac{1}{\Gamma(v+1)}+2 m\left(\mu+v, v+1, \lambda_{1}\right)\right) \vartheta+B\left(\mu, v, \lambda_{1}, \lambda_{2}\right) l_{h}\left(\vartheta^{*}\right) \vartheta^{*}
\end{aligned}
$$


for any $\tilde{x} \in(-\vartheta, \vartheta)^{3}$ and $\eta, \hat{\eta} \in \mathbf{B}_{\vartheta^{*}}(0)$. Since $\vartheta^{*}(1-\rho)=\left(\frac{1}{\Gamma(\nu+1)}+2 m\left(\mu+\nu, \nu+1, \lambda_{1}\right)\right) \vartheta$, we find $\|\mathcal{L P}(\eta)\|_{\infty} \leq \vartheta^{*}$, i.e., $\mathcal{L} \mathcal{P}: \mathbf{B}_{\vartheta^{*}}(0) \rightarrow \mathbf{B}_{\vartheta^{*}}(0)$. The Banach fixed point theorem uniquely determines $\eta_{\tilde{x}}$ by $\eta_{\tilde{x}}=\mathcal{L} \mathcal{P}\left(\eta_{\tilde{x}}\right)$ with $\left\|\eta_{\tilde{x}}\right\|_{\infty}<\vartheta^{*}$. Set

$$
\mathcal{S}(\tilde{x})=-\left(-\lambda_{2}\right)^{\frac{1-\mu}{\mu+\nu}} \int_{0}^{\infty} \exp \left(-\left(-\lambda_{2}\right)^{\frac{1}{\mu+\nu}} s\right) h_{2}\left(\eta_{\tilde{x}}(s), s\right) d s .
$$

Then

$$
|\mathcal{S}(\tilde{x})| \leq\left(-\lambda_{2}\right)^{\frac{1-\mu}{\mu+\nu}} l_{h}\left(\vartheta^{*}\right) \vartheta^{*} \int_{0}^{\infty} \exp \left(-\left(-\lambda_{2}\right)^{\frac{1}{\mu+\nu}} s\right) d s=\left(-\lambda_{2}\right)^{\frac{-\mu}{\mu+\nu}} l_{h}\left(\vartheta^{*}\right) \vartheta^{*}=\vartheta^{* *} .
$$

Furthermore, Proposition 3.3 implies $(\tilde{x}, \mathcal{S}(\tilde{x})) \in W_{0}^{c s}(V \times W, U)$. Clearly (i) holds.

Next we have $\mathcal{L P}=\mathcal{L} \mathcal{P}_{\tilde{x}}$, and in particular $\eta_{\tilde{x}}=\mathcal{L} \mathcal{P}_{\tilde{x}}\left(\eta_{\tilde{x}}\right)$. Then, for any $\tilde{x}=\left(x, x_{3}\right), \tilde{y}=$ $\left(y, y_{3}\right) \in(-\vartheta, \vartheta)^{3}$, it follows from Proposition 3.1 and the definition of $\mathcal{L} \mathcal{P}_{\tilde{x}}$ that

$$
\begin{aligned}
\left\|\eta_{\tilde{x}}-\eta_{\tilde{y}}\right\|_{\infty} & =\left\|\mathcal{L} \mathcal{P}_{\tilde{x}}\left(\eta_{\tilde{x}}\right)-\mathcal{L} \mathcal{P}_{\tilde{y}}\left(\eta_{\tilde{y}}\right)\right\|_{\infty} \\
& \leq\left\|\mathcal{L} \mathcal{P}_{\tilde{x}}\left(\eta_{\tilde{x}}\right)-\mathcal{L} \mathcal{P}_{\tilde{x}}\left(\eta_{\tilde{y}}\right)\right\|_{\infty}+\left\|\mathcal{L} \mathcal{P}_{\tilde{x}}\left(\eta_{\tilde{y}}\right)-\mathcal{L} \mathcal{P}_{\tilde{y}}\left(\eta_{\tilde{y}}\right)\right\|_{\infty} \\
& \leq \rho\left\|\eta_{\tilde{x}}-\eta_{\tilde{y}}\right\|_{\infty}+\left\|\mathcal{L} \mathcal{P}_{\tilde{x}-\tilde{y}}(0)\right\|_{\infty} \\
& \leq \rho\left\|\eta_{\tilde{x}}-\eta_{\tilde{y}}\right\|_{\infty}+\left(\frac{1}{\Gamma(v+1)}+2 m\left(\mu+\nu, v+1, \lambda_{1}\right)\right)\left|x_{3}-y_{3}\right|
\end{aligned}
$$

since $\mathcal{L} \mathcal{P}_{\tilde{x}}(\eta)-\mathcal{L} \mathcal{P}_{\tilde{y}}(\eta)=\mathcal{L} \mathcal{P}_{\tilde{x}-\tilde{y}}(0)$ for any $\eta \in X_{\infty}\left(\mathbb{R}_{+}, \mathbb{R}^{2}\right)$. This yields

$$
\left\|\eta_{\tilde{x}}-\eta_{\tilde{y}}\right\|_{\infty} \leq \frac{1}{1-\rho}\left(\frac{1}{\Gamma(v+1)}+2 m\left(\mu+v, v+1, \lambda_{1}\right)\right)\left|x_{3}-y_{3}\right| .
$$

Hence

$$
\begin{aligned}
|\mathcal{S}(\tilde{x})-\mathcal{S}(\tilde{y})| & \leq\left(-\lambda_{2}\right)^{\frac{1-\mu}{\mu+\nu}} \int_{0}^{\infty} \exp \left(-\left(-\lambda_{2}\right)^{\frac{1}{\mu+\nu}} s\right)\left|h_{2}\left(\eta_{\tilde{x}}(s), s\right)-h_{2}\left(\eta_{\tilde{y}}(s), s\right)\right| d s \\
& \leq\left(-\lambda_{2}\right)^{\frac{1-\mu}{\mu+\nu}} l_{h}\left(\vartheta^{*}\right) \int_{0}^{\infty} \exp \left(-\left(-\lambda_{2}\right)^{\frac{1}{\mu+\nu}} s\right) d s\left\|\eta_{\tilde{x}}-\eta_{\tilde{y}}\right\|_{\infty} \\
& \leq \frac{\vartheta^{* *}}{\vartheta^{*}(1-\rho)}\left(\frac{1}{\Gamma(v+1)}+2 m\left(\mu+v, v+1, \lambda_{1}\right)\right)\left|x_{3}-y_{3}\right|,
\end{aligned}
$$

which gives (ii). The proof is complete.

Finally, we give an example to illustrate our theory.

Example 3.5 Consider the following fractional Langevin equations:

$$
\left\{\begin{array}{l}
{ }^{c} \mathcal{D}_{0, t}^{\frac{1}{2}}\left({ }^{c} \mathcal{D}_{0, t}^{\frac{1}{3}} x_{1}(t)\right)+x_{1}(t)=0, \quad t \geq 0, \\
{ }^{c} \mathcal{D}_{0, t}^{\frac{1}{2}}\left({ }^{c} \mathcal{D}_{0, t}^{\frac{1}{3}} x_{2}(t)\right)-x_{2}(t)=\frac{x_{1}(t)^{2}}{(1+t)^{\frac{2}{5}}}, \\
x(0)=\mathbf{0}=(0,0)^{T}, \quad{ }^{c} \mathcal{D}_{0, t}^{\frac{1}{3}} x(0)=\bar{x}=\left(x_{3}, x_{4}\right)^{T},
\end{array}\right.
$$

where $\left(x_{3}, x_{4}\right)^{T} \in U((0,0), \epsilon), \epsilon$ is an arbitrary positive number. 
Set $\mu=\frac{1}{2}, v=\frac{1}{3}$. Clearly, $\mu+v=\frac{5}{6}<1$. Define $\lambda_{1}=1, \lambda_{2}=-1$ and $h_{1}(x(t), t)=0$, $h_{2}(x(t), t)=\frac{x_{1}(t)^{2}}{(1+t)^{\frac{2}{5}}}, t \geq 0$. Let $l_{h}(\vartheta)=\frac{1}{\imath \vartheta}, \iota>0$ for all $\left\|x_{1}\right\| \leq \vartheta$ and $\left\|x_{2}\right\| \leq \vartheta$. Then (2) holds.

Using (3), the solution $\phi(t, \mathbf{0}, \bar{x})$ is given by

$$
\begin{aligned}
& \phi_{1}(t, \mathbf{0}, \bar{x})=\mathbb{E}_{\frac{5}{6}, \frac{4}{3}}\left(-t^{\frac{5}{6}}\right) t^{\frac{1}{3}} x_{3} \\
& \phi_{2}(t, \mathbf{0}, \bar{x})=\mathbb{E}_{\frac{5}{6}, \frac{4}{3}}\left(t^{\frac{5}{6}}\right) t^{\frac{1}{3}} x_{4}+\int_{0}^{t}(t-s)^{-\frac{1}{6}} \mathbb{E}_{\frac{5}{6}, \frac{5}{6}}\left((t-s)^{\frac{5}{6}}\right) \frac{\phi_{1}(s, \mathbf{0}, \bar{x})^{2}}{(1+s)^{\frac{2}{5}}} d s .
\end{aligned}
$$

If we choose a $\iota>\frac{B\left(\frac{1}{2}, \frac{1}{3}, 1,-1\right)}{\vartheta}$, then $\rho=\frac{1}{\imath \vartheta} B\left(\frac{1}{2}, \frac{1}{3}, 1,-1\right)<1$. In this case, $\lim _{t \rightarrow \infty} \phi(t, \mathbf{0}, \bar{x})=0$.

Hence the Lyapunov-Perron operator $\mathcal{L} \mathcal{P}$ has the form

$$
\begin{aligned}
\mathcal{L P}_{1}(\eta)(t)= & \mathbb{E}_{\frac{5}{6}, \frac{4}{3}}\left(-t^{\frac{5}{6}}\right) t^{\frac{1}{3}} x_{3}, \\
\mathcal{L} \mathcal{P}_{2}(\eta)(t)= & -\mathbb{E}_{\frac{5}{6}, \frac{4}{3}}\left(t^{\frac{5}{6}}\right) t^{\frac{1}{3}} \int_{0}^{\infty} \exp (-s) \frac{\phi_{1}(s, \mathbf{0}, \bar{x})^{2}}{(1+s)^{\frac{2}{5}}} d s \\
& +\int_{0}^{t}(t-s)^{-\frac{1}{6}} \mathbb{E}_{\frac{5}{6}, \frac{5}{6}}(t-s)^{\frac{5}{6}} \frac{\phi_{1}(s, \mathbf{0}, \bar{x})^{2}}{(1+s)^{\frac{2}{5}}} d s .
\end{aligned}
$$

From (10) we derive

$$
\mathcal{S}(\tilde{x})=-\int_{0}^{\infty} \exp (-s) \frac{\phi_{1}(s, \mathbf{0}, \bar{x})^{2}}{(1+s)^{\frac{2}{5}}} d s=-\int_{0}^{\infty} \frac{\exp (-s)}{(1+s)^{\frac{2}{5}}}\left(\mathbb{E}_{\frac{5}{6}, \frac{4}{3}}\left(-s^{\frac{5}{6}}\right) s^{\frac{1}{3}} x_{3}\right)^{2} d s=1.1593 x_{3}^{2} .
$$

Consequently, Proposition 3.3 and Theorem 3.4 imply that the local center stable manifold around the origin is given by $\left\{\left(0,0, x_{3}, 1.1593 x_{3}^{2}\right)\right\}$.

Note that $\left(x_{3}, x_{4}\right)^{T} \in U((0,0), \epsilon)$, and clearly, we have $\lim _{t \rightarrow \infty}\left(\phi_{1}(t, \mathbf{0}, \bar{x}), \phi_{2}(t, \mathbf{0}, \bar{x})\right)=0$ for $x_{3}=0$.

\section{Acknowledgements}

The authors acknowledge the National Natural Science Foundation of China (11661016), Training Object of High Level and Innovative Talents of Guizhou Province ((2016)4006), Unite Foundation of Guizhou Province ([2015]7640), and Graduate ZDKC ([2015]003). The authors are grateful to the referees for their careful reading of the manuscript and their valuable comments.

\section{Competing interests}

The authors declare that they have no competing interests.

\section{Authors' contributions}

All authors have made the same contribution. All authors have read and approved the final manuscript.

\section{Publisher's Note}

Springer Nature remains neutral with regard to jurisdictional claims in published maps and institutional affiliations.

Received: 17 July 2017 Accepted: 3 October 2017 Published online: 18 October 2017

\section{References}

1. Kilbas, AA, Srivastava, HM, Trujillo, JJ: Theory and Applications of Fractional Differential Equations. Elsevier, Amsterdam (2006)

2. Miller, KS, Ross, B: An Introduction to the Fractional Calculus and Differential Equations. Wiley, New York (1993)

3. Podlubny, I: Fractional Differential Equations. Academic Press, San Diego (1999)

4. Tarasov, VE: Fractional Dynamics: Application of Fractional Calculus to Dynamics of Particles, Fields and Media. Springer, Berlin (2011)

5. Zhou, Y, Peng, L: On the time-fractional Navier-Stokes equations. Comput. Math. Appl. 73, 874-891 (2017) 
6. Debbouche, A, Torres, DFM: Sobolev type fractional dynamic equations and optimal multi-integral controls with fractional nonlocal conditions. Fract. Calc. Appl. Anal. 18, 95-121 (2015)

7. Abbas, S, Benchohra, M, Rivero, M, Trujillo, JJ: Existence and stability results for nonlinear fractional order Riemann-Liouville Volterra-Stieltjes quadratic integral equations. Appl. Math. Comput. 247, $319-328$ (2014)

8. Lutz, E: Fractional Langevin equation. Phys. Rev. E 64(051106), 1 (2001)

9. Fa, KS: Generalized Langevin equation with fractional derivative and long-time correlation function. Phys. Rev. E 73(061104), 1 (2006)

10. Picozzi, S, West, B: Fractional Langevin model of memory in financial markets. Phys. Rev. E 66(046118), 1 (2002)

11. Bazzani, A, Bassi, G, Turchetti, G: Diffusion and memory effects for stochastic processes and fractional Langevin equations. Physica A 324, 530-550 (2003)

12. Lim, SC, Li, M, Teo, LP: Langevin equation with two fractional orders. Phys. Lett. A 372, 6309-6320 (2008)

13. Ahmad, B, Nieto, JJ, Alsaedi, A, El-Shahed, M: A study of nonlinear Langevin equation involving two fractional orders in different intervals. Nonlinear Anal., Real World Appl. 13, 599-606 (2012)

14. Wang, J, Fečkan, M, Zhou, Y: Presentation of solutions of impulsive fractional Langevin equations and existence results. Eur. Phys. J. Spec. Top. 222, 1855-1872 (2013)

15. Wang, J, Li, X: Ulam-Hyers stability of fractional Langevin equations. Appl. Math. Comput. 258, 72-83 (2015)

16. Zhao, K: Impulsive boundary value problems for two classes of fractional differential equation with two different Caputo fractional derivatives. Mediterr. J. Math. 13, 1033-1050 (2016)

17. Li, C, Deng, W: Remarks on fractional derivatives. Appl. Math. Comput. 187, 777-784 (2007)

18. Guo, P, Zeng, C, Li, C, Chen, YQ: Numerics for the fractional Langevin equation driven by the fractional Brownian motion. Fract. Calc. Appl. Anal. 16, 123-141 (2013)

19. Li, C, Ma, L: Lyapunov-Schmidt reduction for fractional differential systems. J. Comput. Nonlinear Dyn. 11, 051022 (2016)

20. Li, C, Ma, L: On Hadamard fractional calculus. Fractals 25, 1750033 (2017)

21. Wang, J, Zhou, Y, Wei, W, Xu, H: Nonlocal problems for fractional integrodifferential equations via fractional operators and optimal controls. Comput. Math. Appl. 62, 1427-1441 (2011)

22. Wang, J, Li, X: A uniformed method to Ulam-Hyers stability for some linear fractional equations. Mediterr. J. Math. 13, 625-635 (2016)

23. Wang, J: Approximate mild solutions of fractional stochastic evolution equations in Hilbert spaces. Appl. Math. Comput. 256, 315-323 (2015)

24. Cong, ND, Doan, TS, Siegmund, S, Tuan, HT: On stable manifolds for planar fractional differential equations. Appl. Math. Comput. 226, 157-168 (2014)

25. Sayevand, K, Pichaghchi, K: Successive approximation: a survey on stable manifold of fractional differential systems. Fract. Calc. Appl. Anal. 18, 621-641 (2015)

26. Ma, L, Li, C: Center manifold of fractional dynamical system. J. Comput. Nonlinear Dyn. 11, 021010 (2016)

27. Wang, J, Fečkan, M, Zhou, Y: Center stable manifold for planar fractional damped equations. Appl. Math. Comput. 296, 257-269 (2017)

28. Gorenflo, R, Loutchko, J, Luchko, Y: Computation of the Mittag-Leffler function $E_{\alpha, \beta}(z)$ and its derivative. Fract. Calc. Appl. Anal. 5, 491-518 (2002) Correction: Fract. Calc. Appl. Anal. 6, 111-112 (2003)

29. Peng, S, Wang, J: Existence and Ulam-Hyers stability of ODEs involving two Caputo fractional derivatives. Electron. J. Qual. Theory Differ. Equ. 2015, 52 (2015)

\section{Submit your manuscript to a SpringerOpen ${ }^{\circ}$ journal and benefit from:}

- Convenient online submission

- Rigorous peer review

- Open access: articles freely available online

- High visibility within the field

- Retaining the copyright to your article

Submit your next manuscript at $\gg$ springeropen.com 